\title{
Algunos elementos teóricos para el análisis de políticas exteriores de pequeños paises.
}

\author{
Ethel Abarca Amador.
}

El contexto intemacionaly el Sistema Político interno es el marco global donde se inscribe la política exterior de un pequeño país como Costa Rica.

Este contexto está compuesto por dos tipos de factores: los externos y los internos, Ellos aumentan o disminuyen los márgenes de maniobra de las políticas exteriores de pequeños paises.

Los diversos estudios teóricos que han analizado los elementos que constituyen tanto el ámbito externo como interno de las políticas exteriores de pequeños paises, han subvalorado en alguna medida algunos indicadores internos que inciden en el comportamiento de los márgenes de maniobra.

Esto se manifiesta en la capacidad que tiene un gobernante de elaborar una estrategia de política exterior coherente y viable, asi como que un lider gubernamental cuente con un liderazgo tal, que evite una dispersión en los centros de toma de decisión.

El presente artículo redefine el concepto de «margen de maniobra» y con él sus nuevas características e ilustrarlo en la praxis de las políticas exteriores de las Administraciones Arias Sánchez (1986-1990) y la de Rafael Angel Calderón Foumier (1990-1994).

\section{Redefinición del concepto de "Margen de Maniobra"}

El concepto de "margen de maniobra": ha sido definido de una manera muy amplia y clara por los distinguidos teóricos: Cristina Eguizábal y Francisco Rojas Aravena. Ambos han detallado, en diferentes escritos, los diversos elementos internos y externos que deben tomarse en cuenta para diseñar una Política Exterior de un pequeño país. 
Este paradigma continúa siendo válido y rico para el análisis de las políticas exteriores de los pequeños países. No obstante ello, los profesores Eguizábal y Rojas Aravena reconocen, pero no otorgan suficiente énfasis, al peso específico que algunas variables internas juegan en la definición de los márgenes de maniobra de un pequeño país vis a vis la potencia hegemónica y el sistema internacional en general.

Es necesario, por tanto reformular el concepto de manera tal, que se tomen en cuenta al conjunto de variables domésticas que posibilitan el desarrollo de una política exterior más autónoma. En este sentido, creo conveniente definir el concepto de margen de maniobra de la siguiente manera:

"Entendido como el conjunto de estrategias autónomas que proponga un gobierno ante el contexto externo, especialmente su capacidad para multiplicar los esfuerzos y apoyos internos y externos que tenga a su alcance para lograrlos" (Abarca, 1994; 26).

Esta definición le da mayor énfasis a las variables internas que le otorga la definición tradicional, la cual establece que márgenes de maniobra se define de la siguiente manera:

"Entendidos como el conjunto de opciones que le ofrecen a un gobierno en una problemática dada, especialmente en las posibilidades de multiplicar esas opciones y llevarlas a la práctica" (Eguizábal, 1986: 106).

Ahora bien, debemos dejar cierto claro por qué usamos el concepto de margen de maniobra y no el de margen de autonomía, Ello lo hacemos con base en la perspectiva que al respecto hace de ella Cristina Eguízábal.

La relación que han tenido los países centroamericanos con Estados Unidos, ha sido tradicionalmente una actitud de mayor pasividad, y por ello de menor autonomía; de ahí que usar el término autonomía resultaría inadecuado. Por tanto, aconseja que es más útil trabajar con la noción menos ambiciosa de margen de maniobra, supra citado. En este sentido, el problema de una mayor o menor autonomía no resulta tan central en el análisis y sí el de las posibilidades de multiplicar las opciones y el de la capacidad de hacerlo.

Siguiendo esta misma línea de pensamiento, referente a los elementos que contraen o aumentan los márgenes de maniobra, hay una serie de supuestos, que permiten vislumbrar algunos de los condicionamientos a los cuales están sujetos los márgenes de maniobra de un pequeño país como Costa Rica. 


\section{Estos son:}

1.- En el marco de una condición de dependencia estructural, determinada por su situación de pequeño país, ubicado en la periferia de Estados Unidos, los márgenes de maniobra de que disponen los países centroamericanos en la conducción de su política exterior van a depender de: A) Del tipo de proyecto político interno (o estrategia de desarrollo) que impulsan sus élites; B) Del consenso existente a nivel nacional con respecto a él.

2.- Las agendas de política exterior van a estar, en gran medida, prefijadas por las interacciones regionales, producto de los procesos; internos en cada uno de los países del istmo y por la política del gobierno de Estados Unidos, y finalmente;

3.- La eficiencia y eficacia de la política exterior va a estar condicionada, tanto por el lugar que ocupa el diseño de política internacional, dentro del marco general del proyecto interno, como por medios con los que cuenta para llevar a cabos sus objetivos.

Por otra parte, Francisco Rojas Aravena nos complementa la definición de margen de maniobra cuando apunta que,

"para pequeños países como los ubicados en la región centroamericana, la posibilidad de maniobrar adecuadamente frente a otros actores, dependerá del desarrollo de tres procesos correlativos" (Rojas, 1972; 52).

Estos procesos son, el grado de consenso interno alcanzado para la ejecución de la política. el empleo adecuado de los recursos de poder, por limitados que éstos sean, básicamente la voluntad de usarlos; y finalmente la búsqueda de desarrollo de nuevos apoyos internacionales, tanto gubernamentales como no gubernamentales.

Consideramos válidas estas precisiones. sin embargo, este ensayo revela nuevos condicionamientos, variables y ventajas metodológicas.

En primera instancia, evidencia que el factor principal para que un país pequeño aumente o disminuya sus márgenes de maniobra, residirá en la capacidad que tenga el país para elaborar una estrategia autónoma frente al contexto externo, y no en la posibilidad de multiplicar las opciones que tenga el país en ese contexto. En ese sentido, resulta central en el análisis, el peso que puedan tener las variables internas en las variaciones que tenga el comportamiento de los márgenes de maniobra.

Podemos decir que un país pequeño que logra diseñar una estrategia 
autónoma, a pesar de los condicionamientos externos que se le impongan, es porque aprovechó en forma acertada sus condiciones internas.

Esto nos permite afirmar, por lo tanto, que no sólo es el contexto externo el factor de mayor peso, como lo exponía Cristina Eguizábal en su interpretación, sino un balance entre las variables internas y externas, 1o que define en última instancia el menor o mayor margen de maniobra de un pequeño país. Dado que lo interno es lo que está directamente relacionado con la capacidad de los artífices de la política exterior, es aquí donde se debe poner el énfasis en la formulación de la estrategia; ello, por supuesto, a partir de la lectura que se le haga del contexto externo.

A la luz de esta premisa, se hace necesario redefinir las nuevas variables que deben ser tomadas en cuenta a la hora de definir el mayor o menor margen de maniobra que pueda ser desarrollado por un país pequeño. Algunas de las variables internas de mayor peso, en la elaboración y ejecución de una política exterior de alto perfil, serán el liderazgo presidencial y la elaboración de una estrategia coherente y viable.

Con estos dos nuevos elementos, que contraen o aumentan los márgenes de maniobra, se hace indispensable redefinir las condiciones a las cuales están sujetos dichos márgenes, para un país pequeño como lo es Costa Rica Ellos son:

1.- El tipo de estrategia interna de política exterior que impulse el país, y el consenso existente a nivel de equipo, con respecto a esa estrategia.

2.- La factibilidad de elaborar una estrategia autónoma ante el contexto externo, pasando a ser las interacciones regionales en un aspecto secundario a tomar en cuenta en las agendas de política exterior, y no en el elemento central que determina sus prioridades.

3.- La eficiencia y eficacia de la política exterior, va a estar condicionada por la personalidad y liderazgo que caractericen al gobernante para implementar la estrategia elaborada.

De esto se desprende que las posibilidades que tiene un país pequeño para ampliar sus márgenes de maniobra son reales. De hecho, los logros obtenidos por la Administración Arias Sánchez, demuestran la efectividad que tienen las variables internas en el aumento de los márgenes de maniobra; y por ende, en el desarrollo de la política exterior exitosa, 


\section{Análisis de las Políticas Exteriores de las Administraciones Arias Sánchez y Calderón Fournier, en el indicador de los Márgenes de Maniobra}

Este análisis se basa en un contraste entro las dos políticas exteriores desarrolladas por cada Administración. Para ello se procedió a examinar la existencia de un líder, y la permanencia de una coherencia entre el equipo político y económico en el desarrollo de cada una de las políticas exteriores.

La política exterior de la Administración Arias Sánchez tuvo por énfasis el ámbito político. Sin embargo, en todas las acciones que realizó para ejecutar su estrategia, logró mantener una coherencia entre el equipo político y económico, ello porque se contó con una figura presidencial de amplio liderazgo, que unificó los lineamientos y acciones que deberían llevarse para concretar la política exterior en un resultado real.

Ello se confirma con la teoría de la génesis de las decisiones planteadas por Snayder y otros, la cual nos dice que la existencia de un actor-decisor (líder presidencial) en la política exterior de un pequeño país permite que se unifiquen las acciones de otros actores, así como los fines y los medios que son necesarios para articular los objetivos de una estrategia de política exterior concreta,

Además, este actor decisor (Oscar Arias) definirá la situación en torno al conocimiento que éste tenga en una articulación de las otras condiciones y los otros actores, la existencia o determinación de fines, la asignación de un valor a las diversas líneas de acción sugeridas por la situación, y la limitación del número de alternativas, campo de percepciones y objetivos deseados.

Todo lo cual va a depender de sus actitudes y preparación, ya que estas dos características son las que permiten al actor(Oscar Arias)definir la situación. Su liderazgo presidencial le permite orientar su acción con respecto a la visión no sólo de su interés, sino de la visión que de la situación concreta tienen los otros funcionarios participantes en la estrategia de política exterior.

De todo ello siguiendo a Russel, algo de particular relevancia para este ensayo, es lo siguiente: En la medida en que exista un mayor liderazgo presidencial, en esa medida existirá una menor dispersión en los centros de toma de decisiones. 
En esta Administración el Dr. Oscar Arias Sánchez se constituye en un actor-decisor capaz de evitar la dispersión en los centros de toma de decisión. Ello por las siguientes razones: la cualidad de líder que caracterizó este gobernante y las ideas que éste presenta a lo largo de su trayectoria política, las cuales son producto de su excelente preparación académica, de su espíritu reflexivo, de su profundo sentimiento democrático-pacifista y de su vasta experiencia en la función pública. Sus variados conocimientos en el campo del Derecho, la Economía y las Ciencias Políticas, le han permitido contar con una visión de mundo amplia. Además de ello, conserva su experiencia política durante las cuales fue Ministro de Planificación y Política Económica; asimismo desempeñó el cargo de Diputado y Secretario General del Partido Liberación Nacional (PLN).

También desarrolló un ideario político que no se agota en los asuntos nacionales, sino trasciende el ámbito mundial, y en particular el campo de los problemas centroamericanos. Todo ello le permite tener una gran capacidad de liderazgo en los diversos ámbitos en que participa.

Fue así como a pesar de no estar dentro de la nómina de los favoritos del PLN de 1996, para ostentar la presidencia de la República el 2 de febrero del mismo año, el pueblo lo eligió Presidente de la República con una amplia mayoría de sufragios. Hechos que nos permiten decir que el Dr. Arias desplegó un liderazgo presidencial fuerte y claro durante su labor como gobernante. Lo cual significa una variable interna de carácter relevante para el logro de una política exterior eficaz.

Así lo refleja el Plan de Paz. El Dr. Oscar Arias Sánchez percibió las oportunidades que se generaban tanto en la región como fuera de ella, para desarrollar una política que teniendo presente sus objetivos nacionales fuese capaz de transformarse en el elemento articulador de la paz en la región.

La iniciativa de elaborar una propuesta autónoma en política exterior, a la región centroamericana, será una de las variables que le permita a Costa Rica en esta Administración desarrollar no sólo una política exterior con altos índices de autonomía sino evidenciar la importancia que tiene un líder presidencial como lo fue Oscar Arias para unificar, articular y llevar a cabo con la ayuda de otros actores inmersos en esta tarea una acción concreta en el contexto externo que posibilite el ensanchamiento de los campos de acción con que cuentan los países sometidos a constreñimientos estructurales como es Costa Rica. 
Por otra parte, el presidente aprovechó las condiciones externas en forma apropiada para llevar a concretar su iniciativa en política exterior. A pesar de elaborar una iniciativa a nivel interno concreta, necesitó de las circunstancias internacionales para que el Plan de Paz fuera un éxito.

El Plan de Paz posibilitó, entre otras cosas, que una potencia como lo es Estados Unidos no sólo respetara la posición de Costa Rica frente a la solución del conflicto nicaragüense, sino que al final fuera avalada por este país como la mejor solución, desechando con ello su propia propuesta a tal conflicto. Este hecho, consecuentemente produjo un aumento considerable en los márgenes de maniobra de la política exterior costarricense y un viraje de ciento ochenta grados al rumbo que hasta entonces había tomado la historia centroamericana, a la vez que se pudo lograr una mayor autonomía en la región centroamericana y la capacidad que tuvo un país pequeño de elaborar su propia política exterior.

Al igual que el Plan de Paz, la Renegociación de la Deuda Externa en esta Administración fue una opción positiva para incrementar los márgenes de maniobra.

A pesar de ser la Renegociación de la Deuda Externa la acción más valiosa desplegada por el Dr. Oscar Arias Sánchez en la dimensión económica, ésta necesitó el equipo político para llegar a concretarse.

Ello indica que a lo largo de todas las negociaciones que se realizaron para renegociarse la deuda de Costa Rica, la coherencia éntre lo político y lo económico era lo que permitía al final articular en acciones concretas lo que manejaba el equipo económico.

La Renegociación de la Deuda Externa refleja un incremento en la diversificación de vínculos económicos, ocasionando con ello un aumento satisfactorio en los márgenes de maniobra de la política exterior costarricense.

El Plan de Paz como la Renegociación de la deuda externa fueron las dos iniciativas de mayor significado en la Administración del Dr. Oscar Arias Sánchez. Ambas acciones las realizó el Presidente en un contexto internacional en donde lo política tendía a estar por encima de lo económico. A pesar de ello, al existir poca dispersión en el proceso decisorio de política exterior, amén de un liderazgo presidencial apropiado, se generó una coherencia entre el equipo político y el equipo económico, lo cual es de vital importancia a la luz de los dos objetivos centrales de política exterior. 
La estrategia de política exterior que desarrolló la Administración Arias Sánchez contó con una articulación lógica interna que le permitió al final concretar en hechos reales lo que plasmó en un principio en el papel. Las dos iniciativas de mayor relevancia para la política exterior de la Administración Arias Sánchez, el Plan de Paz y la Renegociación de la Deuda Externa, se fundamentaron en un mismo concepto articulador el Desarrollo.

En el mismo momento que el Presidente estaba negociando en los Estados Unidos la aprobación del Plan en Centroamérica, en el campo económico se estaba negociando en forma simultánea con el Fondo Monetario Internacional, con el Banco Mundial y con los Bancos Privados acreedores, lo que posibilitó que la ayuda norteamericana continuara llegando al país, y con ello, se alcanzara renegociar la deuda externa con los bancos comerciales.

Ello fue una característica constante en cada una de las acciones realizadas por esta Administración, así lo manifestaba el Presidente cada vez que tenía oportunidad de realizar un discurso.

Esto se debió al hecho de que en esta Administración primaron dos premisas básicas sobre las cuales se desarrolló la política exterior.

A.- No puede haber desarrollo sin Paz.

B.- No puede existir una paz verdadera y permanente sin democracia.

El desarrollo, corno el concepto articulador fundamental de los ejes de política exterior, se hizo patente en los diversos discursos que pronunció el Presidente Arias, y en los cuales se deja entrever que Costa Rica cumpliría sus compromisos internacionales siempre y cuando se hicieran ajustes en las reglas del juego. Para ello, debía dársele a los países con deudas elevadas la oportunidad de pagar para alcanzar el desarrollo y por ende la estabilidad política del país y la Región.

En el mismo sentido la paz se transformó en la variable fundamental para la estabilidad del sistema político, el desarrollo económico y social, e incluso para el ejercicio de la autonomía política.

Para Arias, a los países centroamericanos no se los estaba dando el trato preferencial que necesitaban, ya que consideraba insuficientes los acuerdos especiales para vender nuestros productos y pagar nuestras deudas. Se planteó que este comportamiento, por parte de algunos Organismos Internacionales, era un claro obstáculo a los esfuerzos de paz, y que por tanto, era necesario superar esos obstáculos económicos para lograr el Desarrollo y por ende la paz en la región. 
Esto nos indica que hubo un líder que fue capaz de crear un proyecto coherente en política exterior.

Estas ideas nos ilustran cómo evidentemente los esfuerzos realizados por esta Administración en política exterior, tanto a nivel político como a nivel económico, tenían por objetivo conseguir el desarrollo del país.

Todo ello posibilitó que la estrategia no sólo fuera coherente sino también viable; y que por tanto, se le calificara a la política exterior de alto perfil.

A diferencia de la política exterior desarrollada por la Administración Arias Sánchez, la Administración Calderón Fournier desplegó una política exterior que tuvo por énfasis el ámbito económico.

Al contrario de su homólogo, éste no logró una coherencia entre el equipo económico y el equipo político. Ello porque no se contó con un líder presidencial que unificara los objetivos de política y acciones en política exterior por un mismo cause.

Basándonos en la teoría de la génesis de las decisiones planteada por Snayder y otros, Rafael Angel Calderón no asumió su rol de líder presidencial en forma apropiada, ya que estos autores plantean que cuando exista un actor-decisor o líder presidencial en una política exterior de un pequeño país, es posible unificar las acciones de otros actores, los fines y los medios que son necesarios para articular los objetivos de una estrategia de política exterior en acciones reales.

Asimismo este actor-decisor posee una visión del mundo compuesta por las distintas percepciones de los funcionarios participantes en la política exterior.

La ausencia de un líder presidencial en la Administración Calderón Fournier se debe, entre otras, a la personalidad del gobernante. Rafael Angel Calderón ha sido un político tradicional, estudioso del Derecho, con experiencia en la función política y con algún grado de popularidad en el medio político, por ser su padre uno de los más importantes políticos de la década de los cincuenta.

A pesar de contar con una amplia popularidad por ser su apellido, necesitó de la República. Todo ello ha limitado la opinión que este gobernante pueda tener de su visión de mundo y de sus posibilidades de actuar en el contexto externo; factores que nos permiten decir que el Lic. Rafael Angel Calderón Fournier no ha ejercido un liderazgo internacional en su Administración.

Como consecuencia de ello, se presentó en este gobierno una mayor dispersión en los centros de toma de decisión. 
Todo esto conllevó a que en esta Administración existiera una incoherencia entre el equipo económico y el equipo político. Dadas las circunstancias externas las mayores decisiones fueron tomadas por el equipo económico y con ello se desplazó en cierta medida el papel fundamental que tuvo el equipo político y particularmente la Cancillería.

Dado que la tendencia mundial apuntaba hacia la conformación de bloques económicos, y por ende la inserción de pequeños países al libre comercio, los esfuerzos mayores en el campo de la política exterior durante esta Administración estuvieron en manos del Ministro de Comercio Exterior, el Ministerio de Economía y el BCCR.

Ello relegó el papel primordial de la Cancillería en el desarrollo de la política exterior.

Esta centralización de decisiones en el mundo económico estuvo asociada a la relativa solución de la crisis centroamericana, lograda en la anterior administración y al nuevo contexto de integración económica en este gobierno, ocasionando con esto que el factor política quedará en un segundo plano

Esto se ve reflejado en el poco éxito. que tuvieron en esta Administración, la candidatura de Bernd Niehaus a la Secretaría General de la Organización de Estados Americanos (OEA) y la Proclama de un nuevo Orden Ecológico de Cooperación Internacional (NOEI).

Ambas acciones evidenciaron errores importantes en la política exterior desarrollada, y por tanto una disminución en los márgenes de maniobra.

Por un lado, el lanzamiento del Canciller Bernd Niehaus a la Secretaría General de la OEA significó en alguna medida, un factor negativo para la política exterior de ese período. Ello porque la Administración careció de los recursos necesarios para salir adelante en la promoción de una candidatura de tal importancia a nivel regional e internacional. Asimismo, porque el candidato propuesto carecía de una serie de características personales que lo ponían en desventaja con respecto a algunos de sus otros contrincantes,

Por tanto, la iniciativa de elaborar esta propuesta como un objetivo central de la estrategia de política exterior, le restó a esta Administración una posibilidad de ensanchar sus limitados campos de acción, al evidenciar ésta la ausencia de un líder presidencial que unificara, articulara y llevara a cabo, con la ayuda de otros actores inmersos en esta tarea el cumplimiento del objetivo propuesto. 
Además, los factores negativos que se desarrollaron alrededor de la Proclama del NOEI evidencian también una disminución en los márgenes de maniobra producto de la dispersión en la toma de decisiones que existió en esta iniciativa, lo cual provocó que los objetivos de política planteados no se convirtieran en acciones concretas.

Ello muestra, al mismo tiempo, que esta Propuesta a pesar de ser un eje central en la política exterior de este gobierno, tuvo al final un valor marginal para el país y por tanto un alcance mucho menor a nivel internacional.

Al Igual que el lanzamiento del Canciller Bernd Niehaus a la Secretaría de la OEA, la Proclama del NOEI, las Políticas de Apertura Comercial en esta Administración reflejan aspectos negativos de la política exterior, con excepción de la firma del Tratado de Libre Comercio con México.

Las políticas de apertura comercial significaron para el presidente Rafael Angel Calderón, los pilares más relevantes de la política económica exterior que desarrolló su gobierno. Sin embargo, ésta administración le restó importancia al papel fundamental que jugaría el equipo político en la definición de estas propuestas. Esto queda plasmado en la centralización de la toma de decisiones que desplegó el Ministerio de Comercio Exterior y de Economía a la hora de llevar a cabo cada una de las negociaciones internacionales concernientes a este ámbito, relegando a un segundo plano al equipo político.

Por estos motivos tanto la candidatura débil del Canciller Bernd Niehaus a la Secretaría General de la OEA la proclama del NOEI, así como las Políticas de Apertura Comercial, fueran las tres iniciativas de mayor significado en la Administración Calderón-Fournier. Estas tres acciones las realizó el Presidente en un contexto en donde lo económico estaba por encima de lo político. Este no logró en algunas ocasiones, coherencia entre los lineamientos que generaba el equipo económico junto con el político, ocasionando con ello una disminución en los márgenes de maniobra.

La estrategia de política exterior que desarrolló esta Administración careció de una articulación lógica interna entre los ejes centrales de política exterior, a saber. Secretaría General de la OEA, la Proclama de un Nuevo Orden Ecológico de Cooperación Internacional y las Políticas de Apertura Comercial. Ello evidencia que no existió un sólo propósito de política exterior, Más bien, por el contrario, la estrategia expone cada uno de los ejes como temas aparte, sin ningún tipo de ligamen unos con los otros, lo 
cual consecuentemente, produce una incoherencia entro los planteamientos y las acciones a seguir.

Esta desarticulación provocó una fragmentación en los entes decidores, los cuales diseñaron sus políticas en forma individual, sin tomar en cuenta los otros objetivos que conforman la estrategia, de los cuales ellos eran solo una parte.

Ello conlleva, por tanto, a que la política exterior desarrollada por esta Administración sea calificada como una política exterior de bajo perfil.

\section{Consideraciones finales}

Los elementos teóricos para el análisis de políticas exteriores de pequeños países se han caracterizado por dar mayor énfasis al contexto internacional en donde se inscribe esta política, que al Sistema Político interno donde se formulan los lineamientos a seguir en el campo de las Relaciones Internacionales. Al respecto, este ensayo llega a dos grandes conclusiones.

- Un país pequeño, a pesar de sus limitantes geográficas, económicas y de poder, puede diseñar una estrategia de política exterior que lo permita incrementar sus campos de acción a un nivel deseable, con el cual obtener un mayor margen de maniobra en sus políticas exteriores.

- Una estrategia de política exterior de un país pequeño debe contar con un líder gubernamental, que evite una dispersión en los centros de toma de decisión, para lograr unificar los objetivos que se han estipulado en el Plan de Acción de la política, sino existe un actor estatal que articule los ejes de la política exterior, existirá una fragmentación en Ja estrategia planteada.

Por lo que existe un actor gubernamental, que articule la estrategia, los márgenes de maniobra disminuye.

Para próximas investigaciones en el campo del análisis de políticas exteriores de pequeños países, sería de mucha utilidad analizar con mayor profundidad las variables internas, para de ese modo dilucidar que posibilidades "reales" tiene un país pequeño para aumentar o disminuir sus márgenes de maniobra; y con ello desdibujar la premisa de que es el contexto externo el que determina nuestras políticas exteriores. 
Bibliografía:

Ethel Abarca Amador:

Algunos elementos teóricos para el análisis de políticas exteriores de pequeños paises.

Abarca, Ethel. La Politica Exterior Costarricense a la Luz de los condicionamientos del Sistema Internacional (1986-1992). Tesis de Licenciatura en Ciencias Políticas, Universidad de Costa Rica, San Pedro, 1994

La Política Exterior de la Administración Calderón Fournier ¿ Reducción de los márgenes de maniobra o Incorporación al nuevo contexto internacional? En: Revista de Ciencias Sociales, número 70:57-67, diciembre de 1995.

Aguilar, Marianela. La Estrategia y estilo de la política exterior. Plan de Paz y Renegociación de la deuda externa, Administración Arias Sánchez (1996-1990) Universidad de Costa Rica, Instituto de Investigaciones Sociales (IIS), San Pedro 1991.

Benavides, Tatiana. El impacto de las negociaciones de paz en Centroamérica (1978. 1988 sobre la política exterior costarricense hacia la región. Tesis de Licenciatura en Ciencias Políticas, Universidad de Costa Rica, San Pedro 1993.

Bustamante, Fernando. Política Exterior de la Unión hacia América Latina: La hora de la renegociación, En: Heraldo Muñoz (comp.). Las Políticas Exteriores de América Latina y el Caribe: Un balance de Esperanzas. Anuario de Políticas Exteriores Latinoamericanas, Grupo editor Latinoamericano (GEL), Buenos Aires, 1988.

East, Maurice. Perspectivas para el estudio de la autonomía de pequeños estados en el Sistema Internacional. En: Revista de Estudios Sociales Centroamericanos, número 95 , San José, enero-abril de 1987.
Eguizábal, Cristina. América Latina y la crisis Centroamericana. En busca de una solución regional. Grupo Editor Latinoamericano, Buenos Aires, Argentina 1987.

Las políticas Exteriores Centroamericanas frente a la crisis regional. Elementos para una Comparación En: América Latina y la crisis Centroamericana. En busca de una solución regional. Grupo Editor Latinoamericano, Buenos Aires, 1988.

Eguizábal, Cristina y Araya, Andrés. Una aproximación al Estudio de las Políticas Exteriores Centroamericanas. En: Revista de Estudios Sociales Centroamericanos, número 43 San Pedro, Costa Rica, enero-abril, 1992.

Eguizábal, Cristina y Aguilar, Marianela. Procesos de Decisión de Política Exterior de Costa Rica: Administración Monge Alvarez 1982-1986. Avance de Investigación, número 75, IIS, Universidad de Costa Rica 1990.

Eguizábal, Cristina et al, La Administración Carazo Odlo como período de transición hacia un nuevo estilo de hacer política exterior. Contribución, número 9, IIS, UCR, mayo, 1991.

Entre la Alianza y la Crisis: Administración Monge ALvarez (mayo 1982-nov 1983). Serie de Documentos \# 2B IIS, UCR. 1990.

A la búsqueda de un mayor margen de acción: Administración Monge Alyarez (nov 1983- oct 1984). Serie de Documentos \#2,B, IIS, UCR. 1990.

La Nueva Estrategia-Administración Monge Alvarez (oct. 1984-mayo 1986).

Ministerio de Relaciones Exteriores y Culto. Memoria Anual 1990-1991. 1 de mayo de 1991.

Memoria Anual 1992-1993. Asamblea Legislativa. 1 de mayo de 1993.

Rojas Aravena, Francisco. Esquipulas II: El Desarrollo de la Paz. En: Revista Relaciones Internacionales, \#26, UNA, Heredia, Costa Rica, Primer Trimestre, 1989. 
Política Exterior de la Administración Arias. Sánchez 1986-1990. UNA, Heredia, 1990.

\begin{abstract}
Acuerdos Políticos y Conflictos Centroamericanos, En: Revista del Consejo Latinoamericano, Integración Política y cuestión nacional en América Latina. Año XIX, abril de 1990.
\end{abstract}

Costa Rica y Honduras: Similares Problemas, Soluciones Distintas. En: Revista de Estudios Sociales Centroamericanos. CSUCA, \#43, enero-abril de 1979.

Russell, Roberto. Polfitica Exterior y toma de decisiones en América Latina: Aspectos Comparativos y Consideraciones Tebricas. En: Cristina Eguizábal y Francisco Rojas: Política Exterior y procesos de decisión en Centroamérica: Elementos para una aproximación a los procesos de negociación regional. Anuario de Estudios Centroamericanos, 1989.

Solís, Luis Guillermo. "Costa Rica: La Politica Exterior en el Sistema Internacional en los años ochenta". En: Juan Manuel Villasuso (comp.). En Nuevo Rostro de Costa Rica. CEPAL, Heredia, 1992.

Tomassini, Luciano. "Introducción a la Teoria de las Relaciones Internacionales". En: Cuadernos de Ciencias Sociales, $\mathrm{N}^{\circ} 25$, FLACSO, San José, abril 1988.

Yan Klaveren, Alberto. "El análisis de la política exterior latinoamericana: Perspectivas teóricas". En: Heraldo Muñoz y J. Tulchin: Entre la autonomía y la subordinación de los países latinoamericanos. Buenos Aires, GEL, 1984. 